\title{
Échanges humains et culturels en Méditerranée dans les manuels scolaires, un thème d'actualité
}

Sylvie Cromer et Mostafa Hassani-Idrissi

\section{(2) OpenEdition}

Journals

Édition électronique

URL : http://journals.openedition.org/trema/2596

DOI : $10.4000 /$ trema.2596

ISSN : 2107-0997

Éditeur

Faculté d'Éducation de l'université de Montpellier

Édition imprimée

Date de publication : 1 décembre 2011

Pagination : $1-7$

ISSN : 1167-315X

Référence électronique

Sylvie Cromer et Mostafa Hassani-Idrissi, «Échanges humains et culturels en Méditerranée dans les manuels scolaires, un thème d'actualité », Tréma [En ligne], 35 - 36 | 2011, mis en ligne le 22 mars

2012, consulté le 22 septembre 2020. URL : http://journals.openedition.org/trema/2596 ; DOI : https:// doi.org/10.4000/trema.2596

Ce document a été généré automatiquement le 22 septembre 2020.

Trema 


\title{
Échanges humains et culturels en Méditerranée dans les manuels scolaires, un thème d'actualité
}

\author{
Sylvie Cromer et Mostafa Hassani-Idrissi
}

1 Du 12 au 14 novembre 2009, se tenait le colloque international et interdisciplinaire Échanges humains et culturels en Méditerranée dans les manuels scolaires à l'université Paul Valéry-Montpellier III (France), rassemblant 70 chercheur-e-s de toutes disciplines (histoire, didactique, analyse du discours, anthropologie, sociologie, biologie...) et de tout l'espace euro-méditerranéen (21 pays au total). L'objet du colloque était de repérer comment les manuels scolaires reflètent ou induisent des échanges entre groupes humains, nations et cultures, et ainsi construisent et véhiculent des représentations de Soi et de l'Autre. La publication des actes de ce colloque ${ }^{1}$ coïncide avec la récente actualité. Les Indignés européens faisant écho aux «printemps arabes » du Maghreb au Machreq attestent de la réalité des échanges culturels dans le pourtour méditerranéen, échanges accélérés par la mondialisation et la technologie, mais aussi sous-tendus par la généralisation de l'éducation des filles et des garçons, c'est-à-dire la circulation des savoirs et des valeurs.

Les dix communications rassemblées par le présent numéro de Tréma interrogent justement les manuels scolaires à l'aune des valeurs démocratiques prônées généralement dans les discours officiels et notamment dans les orientations des ministères de l'éducation nationale: de quelles valeurs ces manuels sont-ils en fait porteurs, implicitement ou explicitement?

\section{Une question récurrente, renouvelée par de nouvelles méthodes d'analyse}

Mais ne serait-ce pas une question rebattue, de surcroît aux réponses évidentes, et donc intellectuellement stérile, que celles des valeurs, comme le prétendait en 1984 Pierre CASPARD? 
3 Rebattue certes, puisque, dès 1925 la Société des Nations propose une analyse comparée des manuels pour combattre la xénophobie et le racisme, relayée en 1946 par l'Unesco. Dans les années 1970, ce sont les stéréotypes liés au sexe qui sont remis en cause et objet d'un programme d'études nationales initié dans le sillage de la Conférence mondiale de la décennie des Nations Unies pour la femme de Copenhague 1980 (MICHEL 1986). La question des préjugés dans les manuels scolaires et autres matériels didactiques garde toute son actualité, renouvelée par la promotion d'une éducation fondée sur les droits humains. En tant qu'organisateur des connaissances dans une société, le manuel est investi comme un instrument majeur pour introduire un processus de changement social (BRUGEILLES et CROMER, 2008). D'autant qu'il reste le moins coûteux des médias, malgré les difficultés non négligeables qui peuvent subsister, dans certaines régions, pour le concevoir, le produire, le diffuser et le mettre à disposition de tous les élèves.

4 Les manuels scolaires ne sont pas de simples supports de transmission de connaissances, ils représentent pour l'éducateur désireux de transmettre des modèles d'assimilation un véhicule privilégié.

Par-delà les faits, les dates et les personnages historiques ou fictifs dont les auteurs ponctuent leur récit, le manuel contient un discours idéologique. À travers les connaissances, mais aussi à travers les opinions sur l'organisation sociale et politique d'un pays, le manuel comporte des appels qui s'adressent à l'élève, lui suggèrent ce qu'il faut aimer et respecter et ce qu'il faut haïr et mépriser. Le manuel participe ainsi à la construction du système de valeurs de l'élève. Il lui transmet des modèles d'identification, lui trace des idéaux et l'oriente, contribuant ainsi au renforcement de son surmoi ${ }^{2}$.

5 Ainsi, au XXIe siècle, un double constat s'impose : l'importance persistante, dans un monde globalisé et multimédiatisé, du manuel, et la nécessité de sa révision permanente pour mieux favoriser les apprentissages comme mieux prendre en compte les valeurs de la société qui l'utilise. En témoignent les controverses et polémiques qui agitent régulièrement l'espace politique et médiatique, au-delà du monde de l'éducation. Ainsi, en 2005, lors de la réédition de manuels d'histoire minimisant les atrocités commises par l'armée impériale, notamment le massacre de Nankin en 1937, des manifestations anti-japonaises se déclenchèrent en Chine et en Corée du Nord. En 2005 encore, on assista à la levée de boucliers soulevée par la loi française du 23 février 2005 « portant reconnaissance de la Nation et contribution nationale en faveur des Français rapatriés", dont l'article 4 stipulait que "Les programmes scolaires reconnaissent en particulier le rôle positif de la présence française outre-mer, notamment en Afrique du Nord, et accordent à l'histoire et aux sacrifices des combattants de l'armée française issus de ces territoires la place éminente à laquelle ils ont droit ». Tout récemment, la rentrée scolaire française de 2011 a été à son tour agitée par les pétitions de députés et de sénateurs, sommant l'État d'intervenir, suite à la prise en compte du genre dans les programmes officiels et dans quelques manuels de SVT...

Connaissances et valeurs sont bien intrinsèquement liées, au cœur de ce «bien commun ", de toutes les sociétés qu'est le manuel, miroir symbolique de la construction et de l'expression de l'image de soi et de l'autre, à titre collectif comme à titre individuel. Or les définitions identitaires, malgré qu'on en ait, sont plurielles et complexes, avec le poids de l'histoire, la mondialisation de la communication, la diversification des instances de socialisation... Et les études ici présentées montrent que 
le didactisme, censé supposer clarifier les intentions, ne peut dépasser les enjeux contradictoires dont est porteur le manuel : éducatif, culturel, idéologique. Le système sous-jacent de valeurs des manuels reste un système paradoxal.

\section{Présentation des articles}

6 L'axe commun des valeurs qui réunit les dix communications du volume est étudié de manière concrète en s'intéressant au contenu des connaissances transmises ou aux représentations. Chaque étude aborde, par différentes disciplines (sociologie, sciences du langage, science de l'éducation, linguistique, histoire) et méthodologies (analyses synchroniques ou diachroniques de contenu et de discours, méthodes quantitatives ou qualitatives, comparatisme), la place de l'Autre, que cet Autre soit l'autre sexe, les minorités ethniques, les autres peuples.

\section{Trois points font l'originalité de ce corpus d'études}

7 1) Huit pays sont concernés: Algérie, Chypre, France, Italie, Liban, Malte, Maroc, Tunisie. Sauf Malte, chacun des pays fait l'objet d'au moins deux articles (par exemple pour l'Algérie, voir LAKHAR-BARKA et EL MESTARI, pour les manuels d'anglais et d'éducation religieuse.

2) Des manuels, de l'enseignement primaire comme de l'enseignement secondaire et de différentes disciplines sont analysés: l'anglais, la biologie, les mathématiques, l'éducation religieuse (en arabe), l'éducation civique, le français, langue première ou langue étrangère, la grammaire, l'histoire, la géographie, les sciences économiques et sociales, montrant ainsi que les manuels d'histoire ne sont pas les seuls vecteurs de représentations et des valeurs. On notera le choix novateur des manuels scientifiques.

3) Les corpus de manuels passés au crible sont souvent impressionnants : 29 manuels pour l'étude de la $\mathrm{HALDE}^{3}$ en France (article de SINIGAGLIA AMADO) ; respectivement 28 et 18 manuels pour deux articles (AGORRAM et al et QUESSADA et al.) écrits dans le cadre du projet européen de recherche Biohead-Citizen (Biology, Health and Environmental Education for better Citizenship), coordonné par G. CARVALHO, P. CLÉMENT et F. BOGNER de 2004-2008.

\section{Trois questionnements se dégagent des articles}

\section{Qu'est-ce que I'humain?}

8 Les deux articles issus du programme de recherche Biohead-Citizen s'intéressent, en s'appuyant au sein des livres de biologie sur les chapitres de la génétique pour l'un (AGORRAM et al.), de l'évolution pour l'autre (QUESSADA et al.) aux interactions entre connaissances scientifiques et systèmes de valeurs. En fonction des contextes (socioéconomique, culturel, religieux, historique...), les connaissances et leur enseignement subissent des variations et sont influencés par des conceptions implicites telles que l'anthropocentrisme, le finalisme, l'occidentalisme, le dogmatisme, l'androcentrisme... toutes conceptions en contradiction avec les valeurs universelles telles que la liberté et l'égalité. 


\section{Quelle image de soi, quelle place à l'Autre?}

Quatre articles s'interrogent sur l'éducation à l'altérité dans quatre pays qui ont connu des conflits civils, voire des tensions intercommunautaires fortes, le Liban, l'Algérie et Chypre. EL MESTARI révèle l'ambiguïté de l'éducation religieuse en Algérie, oscillant entre transmission de connaissances de type socio-historiques sur la religion et production de discours idéologiques, propres à façonner des mentalités ethnocentriques. DENIMAL et OSSEIRAN, à partir des thématiques des manuels de français langue étrangère au Liban, pointent $\mathrm{du}$ doigt le fait que l'ouverture à l'autre s'accompagne de l'occultation de la diversité sociale libanaise. BAIDER et al. s'interrogent, concernant Chypre, sur la place des «subalternes» dans les livres d'histoire où n'est pas prise en compte l'expérience des communautés minoritaires qui restent invisibles, malgré les revendications de reconnaissance. Cet article aborde aussi les lacunes en matière d'égalité des sexes, quelle que soit la communauté : « la nation est encore symbolisée par l'être masculin et le genre masculin est encore en grammaire celui qui peut représenter les deux sexes». Enfin se rattache aussi à cette problématique l'article de SINIGAGLIA-AMADO qui se penche sur le traitement des populations minoritaires, visibles ou invisibles: telles les handicapés, les seniors, les personnes de la « diversité culturelle »... et bien sûr les femmes.

\section{Quelle place pour les femmes et les rapports hommes/femmes?}

À des degrés divers, la question de la représentation des femmes est le fil conducteur de ce volume. Sept textes la prennent en considération et cinq la mettent au cœur de leur problématique.

Nous venons de citer le texte de BAIDER et al. À partir de la grammaire, LAKDHAR-BARKA, dans une étude diachronique fondée sur l'analyse des représentations des femmes dans les textes et les images dans les manuels d'anglais en Algérie, conclut à son " évaporation " au fil du temps, liée au contexte historique. Pour la France, SINIGAGLIAAMADO, dans le cadre du rapport commandé par la HALDE, balaie toutes les disciplines et atteste non seulement de l'absence de diversité de modèles, mais de la présence de stéréotypes que l'on aurait cru dépasser.

Mettant en œuvre une méthodologie commune, basée sur une analyse quantitative des personnages et reposant sur le concept de genre, deux articles recensent de manière exhaustive les personnages des manuels de mathématiques et, à partir des caractéristiques de leur sexe social, «content » les rapports de genre que reflètent les sociétés fictives des manuels. L'article de BоUсноисна et Lосон concerne la Tunisie, celui de BRUGEILles et CROMER, la France. Si dans les deux cas de figure, les représentations sont inégalitaires, le masculin l'emportant numériquement et qualitativement, le système de genre - l'ensemble des normes, croyances, pratiques, connaissances qui construisent la différence des sexes et organisent de manière inégalitaire les rapports entre les hommes et les femmes - n'est pas pour autant le même, attestant dans la fiction comme dans la réalité de la plasticité des rapports sociaux de sexe.

11 Ces articles renvoient à la recommandation du rapport européen Eurydice: «Des orientations explicites pour le développement de manuels scolaires non sexistes et l'évaluation régulière $d u$ matériel pédagogique pourraient sans doute s'avérer utiles pour remédier au manque de sensibilisation à la dimension de genre. » (p.71).

Certes, les stéréotypes, omniprésents dans notre vie quotidienne et au cœur de notre 
façon de penser, ne serait-ce que pour faciliter la communication et l'action, ne sont en aucun cas la cause des inégalités (DESCARRIES et al., 2010), mais ils contribuent à l'altérisation et à la stigmatisation de l'Autre et leurs effets négatifs ont été mis évidence : que ce soient pour les femmes ou pour d'autres catégories. Toutefois nous demeurons persuadés que l'analyse des manuels scolaires ne peut être que partielle. Lui échappent d'autres lectures, ainsi que l'efficacité du contenu communiqué et le commentaire oral dont l'institutrice ou le professeure accompagne la leçon. Des contradictions peuvent être observées tant au niveau de l'émission (il serait hasardeux de croire que les enseignants tiennent un discours monolithique) qu'au niveau de la réception (les élèves de milieux socio-culturels différents, sont déjà porteurs de valeurs différentes).

12 C'est maintenant à un voyage que nous vous convions grâce aux manuels scolaires : un cheminement réflexif pour aiguiser le regard nécessairement critique à l'endroit de nos indispensables manuels.

La géographie documentaire, classe de fin d'études primaires, Louis Panel

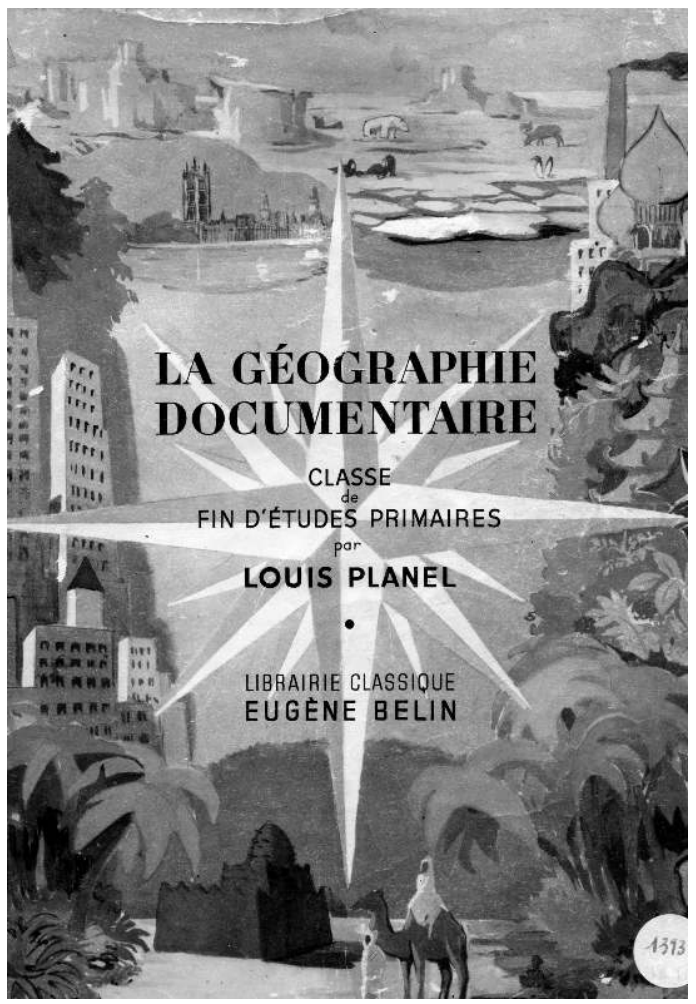

Paris : Librairie Classique Eugène Belin, 1947 - Couverture (Illustration André Chausson). Source CEDRHE. 


\section{BIBLIOGRAPHIE}

BRUGEILLES C., CROMER S., Promouvoir l'égalité entre les sexes par les manuels scolaires. Un guide pour les acteurs et actrices de la chaîne du livre. Unesco, 2008 (téléchargeable sur unesco.doc http:// unesdoc.unesco.org/images/0015/001588/158897f.pdf)

CASPARD P., « De l'horrible danger d'une analyse superficielle des manuels scolaires », revue Histoire de l'éducation $\mathrm{N}^{\circ} 21$, janv. 1984 p.67-74

DESCARRIES F., MATHIEU M., Entre le rose et le bleu. Étude sur les stéréotypes sexuels et construction sociale du féminin et du masculin. Québec ; Conseil du statut de la femme, 2010

MICHEL A., Non aux stéréotypes : vaincre le sexisme dans les manuels scolaires et les livres pour enfants. Paris, UNESCO, 1986

Réseau Eurydice « Éducation, audiovisuel et culture », Différences entre les genres en matière de réussite scolaire : étude sur les mesures prises et la situation actuelle en Europe. (http:// www.eurydice.org), 2010

VERDELHAN-BOURGADE M., BAKHOUCHE B., ETIENNE R., BOUTAN P., Les manuels scolaires, miroirs de la nation ? Paris, L'Harmattan, 2007

\section{NOTES}

1. Pour lire d'autres communications du colloque, cf. Amandine DENIMAL, Arouna DIABATÉ et Michèle VERDELHAN-BOURGADE (coord.), Manuels et altérités dans l'espace méditerranéen. Enjeux institutionnels et linguistiques, Paris, L'Harmattan, 2011

2. ANSART P., "Manuels d'histoire et inculcation du rapport affectif au passé ", dans Enseigner l'histoire. Des manuels à la mémoire, textes réunis et présentés par Henri MONIOT, Berne, Peter LANG, 1984, p. 67.

3. La Haute Autorité de Lutte contre les Discriminations et pour l'Egalité.

\section{AUTEURS}

\section{SYLVIE CROMER}

Université Lille 2, Droits et perspectives du droit Université Lille Nord de France-UDSL, France

\section{MOSTAFA HASSANI-IDRISSI}

Université Mohammed V, Rabat, Maroc 